\title{
Orofacial Clefts: Our Experience in Two Suburban Health Facilities
}

\section{Ogunmuyiwa Stella Aimiede ${ }^{1 *}$, Gbolahan Omoyosola Olalere ${ }^{2}$, Olaosun Adedayo $\mathrm{O}^{3}$ and Sotannde Adeshola I ${ }^{4}$}

${ }^{1}$ Oral and Maxillofacial Surgery Unit, Dental Services Department Federal Medical Centre, Abeokuta, Nigeria

${ }^{2}$ Department of Oral and Maxillofacial Surgery, College of Medicine, University of Ibadan, Nigeria

${ }^{3}$ Department of Otorhinolaryngology, College of Health Sciences, Ladoke Akintola University of Technology, Osogbo, Nigeria

${ }^{4}$ Department of Anaesthesia, Federal Medical Centre, Abeokuta, Nigeria

\begin{abstract}
Background: There may be gross under-reporting of clefts in developing nations due to late and non-presentation The aim of this study was to highlight the descriptive epidemiology of the cleft lip and palate cases managed at the Oral and Maxillofacial Surgery units of two secondary health facilities in southwestern Nigeria and the factors accounting for late presentation.

Methodology: A prospective, cross-sectional study with consecutive recruitment of consenting cleft patients on presentation at the facilities between July 2010 and February 2012. A questionnaire was used to collect patients' data. The data was analyzed using SPSS version 17.0 (SPSS, Chicago, IL, USA) to present descriptive statistics.

Results: Sixty three patients participated: 27 males (42.9\%), 36 females (57.1\%). The median age was 8 years (age range 1 day to 41 years). In addition the age distribution was multimodal (modal ages: 1 year and 4 years). The male to female ratio was 1: 1.3. The most common type of cleft seen was unilateral cleft lip with or without palate $(50.8 \%)$ while median cleft was the least common $(1.6 \%)$. About $9.5 \%$ of the cleft cases were associated with congenital anomalies. Consanguinity was not found in any of the patients. Over $70 \%$ of the patients presented late for treatment with financial constraints being the most common reason for late presentation. Only $6.3 \%$ of the patients gave a family history of clefts. Only a third of those who had surgery were present at the third follow up visit.

Conclusions: Late presentation, poor compliance to follow up and poverty remain the great challenges to OFC repair services in developing countries. It is believed that increasing availability of OFC repair services, together with an increase in awareness campaigns, may help to resolve these issues. There is a need for well designed prospective studies in the developing world to assess the true burden of the entity.
\end{abstract}

Keywords: Orofacial cleft; Poverty; Late presentation; Underreporting; Congenital anomalies

\section{Introduction}

Orofacial Clefts (OFC), including cleft lip with or without palate (CLP) and Cleft Palate (CP) without cleft lip is amongst the most common birth defects of the head and neck. These deformities are cosmetically conspicuous and therefore constitute a serious affliction to the patients and their families [1,2]. Studies suggest that it is multifactorial with interplay between genetic and environmental factors $[1,3,4]$. In addition to these factors, an association between orofacial clefts and consanguinity has been established $[5,6]$. Consanguinity is considered a significant factor in autosomal recessive diseases and has also been associated with congenital anomalies such as hydrocephalus, polydactilia and cleft lip and palate [5]. The risk of congenital conditions is higher in subjects born of first degree consanguineous parents compared with those of non-consanguineous marriages [7]. These factors may have a varying effect in different communities and cultures, and may have a significant influence on the wide variation of the reported incidence.

The epidemiological data in the literature shows the incidence of OFC to be approximately $1 / 600-1 / 1000$ live births. This wide variability in incidence is presumably due to the variation in the geographic and ethnic distribution $[1,8,9]$. Asian populations tend to show the highest prevalence, with Caucasian populations showing intermediate and African populations the lowest prevalence $[9,10]$. Although the epidemiology of orofacial cleft has been extensively studied worldwide [11-13], there may still be gross under-reporting of this condition in several developing nations, especially in the rural and suburban areas. Possible reasons for the under-reporting include poverty, ignorance, cultural beliefs, and lack of access to OFC repair facilities [14,15]. However, with increasing public awareness campaigns, sponsorship for treatment by various charity organizations and increase in the number of centres managing OFC, an increase in the number of cases treated is to be expected.

The main objectives of this study therefore are to highlight our experience and present the descriptive epidemiology of orofacial clefts in two secondary health facilities in south western Nigeria.

\section{Materials and Methods}

A descriptive cross-sectional study was undertaken between July 2010 and February 2012. The study was conducted at the Oral and Maxillofacial units of the Federal Medical Centre (FMC) and Sacred Heart Hospital, both in Abeokuta, southwest Nigeria. All patients who presented with orofacial clefts during the study period and who gave their consent were included in the study. A questionnaire was used to capture demographic, sociocultural, clinical, operative and follow up data.There was an attached explanatory note as to the purpose, voluntary nature and the confidentiality of their participation in the study. An informed consent was obtained from the participants at recruitment into the study.The study was approved by our institutional Ethics committee.

${ }^{*}$ Corresponding author: Dr. Ogunmuyiwa Stella Aimiede, Oral and Maxillofacia Surgery Unit, Dental Services Dept, Federal Medical Centre, Abeokuta, Nigeria, Tel: 2348034503868; E-mail: sheistella@yahoo.com

Received February 22, 2013; Accepted April 10, 2013; Published April 12, 2013

Citation: Aimiede OS, Olalere GO, Olaosun Adedayo O, Sotannde Adeshola I (2013) Orofacial Clefts: Our Experience in Two Suburban Health Facilities. Dentistry 3: 155. doi:10.4172/2161-1122.1000155

Copyright: (ㅇ 2013 Aimiede OS, et al. This is an open-access article distributed under the terms of the Creative Commons Attribution License, which permits unrestricted use, distribution, and reproduction in any medium, provided the original author and source are credited. 
Data on associated congenital anomalies were also collected. Data was analysed using SPSS version 17.0 (SPSS, Chicago, IL, USA) to present descriptive statistics.

\section{Operative Procedure}

Unilateral cleft lip repair was performed using the standard Millard's rotation advancement flap while the bilateral cleft lip defect was repaired using a fork flap technique under general anaesthesia. Von Langenbeck's double palatal flap technique was used for the palatal cleft repair.

\section{Results}

A total of sixty three (63) consecutive patients constituted the study population. The median age was 8.0 years ( 1 day to 41 years) with a male to female ratio of $1: 1.3$. Thirty per cent of the patients were less than a year old. Clinicodemographic data are presented in table 1. Isolated cleft palate showed the highest frequency in females $(27.0 \%$ versus $4.8 \%$ (males)) while the isolated cleft lip showed the highest frequency in males (20.6\% versus $9.5 \%$ (females)). Overall, females were slightly more affected than males among all clefts with a frequency of $46 \%$ and $22.2 \%$ respectively. About two third of all the unilateral cleft cases (65.6\%) were on the left side. Although none of the patients seen in this study gave a history of consanguinity as a possible aetiological factor, a positive family history of cleft was obtained in 4 (6.3\%) of the patients with cleft palate either in isolation or with cleft lip. $3(4.8 \%)$ of the study population were products of multiple gestations (twin pregnancies). The three sets of twins were dizygotic but only one of each

\begin{tabular}{|c|c|c|}
\hline Age range (years) & Number & Percentage \\
\hline$<1$ year & 19 & $(30.2)$ \\
\hline $1-5$ years & 12 & $(19.0)$ \\
\hline $6-10$ years & 4 & $(6.3)$ \\
\hline $11-15$ years & 8 & $(12.7)$ \\
\hline $16-20$ years & 4 & $(6.3)$ \\
\hline$>20$ years & 16 & $(25.4)$ \\
\hline Total & 63 & $(100)$ \\
\hline Gender & & \\
\hline male & 27 & $(42.9)$ \\
\hline female & 36 & $(57.1)$ \\
\hline Total & & $(100)$ \\
\hline Type of cleft deformity & & \\
\hline Unilateral cleft lip \pm palate & 32 & $(50.8)$ \\
\hline Bilateral cleft lip \pm palate & 7 & $(10.1)$ \\
\hline Isolated cleft palate & 20 & $(31.7)$ \\
\hline Median cleft palate & 1 & $(1.6)$ \\
\hline Tessier 7 orofacial cleft & 3 & $(4.8)$ \\
\hline Total & 63 & $(100)$ \\
\hline \multicolumn{2}{|c|}{ Table 1: Clinicodemographic data } \\
\hline
\end{tabular}

Table 1: Clinicodemographic data.

\begin{tabular}{|c|c|c|}
\hline Congenital anomaly & Number & (\%) \\
\hline Congenital heart disease & 1 & 1.6 \\
\hline Low set ears & 1 & 1.6 \\
\hline microcephaly & 1 & 1.6 \\
\hline Skin warts & 1 & 1.6 \\
\hline Syndactyl & 1 & 1.6 \\
\hline Talipase equinovarus & 1 & 1.6 \\
\hline Total & 6 & 9.5 \\
\hline
\end{tabular}

Table 2: Associated congenital anomalies with OFC. set was affected with an orofacial cleft. About 9.5\% of the cleft cases were associated with other congenital anomalies as shown in table 2.

Over $70 \%$ of the patients presented late for treatment with financial constraints being the most common reason (38.1\%). Of the 43 (68.3\%) patients who had previously presented to other health care facilities for treatment, only $9.5 \%$ had cleft lip and palate repair done at the initial facility where they had earlier presented. Thirty seven (58.7\%) of these patients received no treatment mainly due to financial constraints.

While about $70 \%$ of the total patients seen during the study had surgery done, four patients $(6.3 \%)$ declined surgery and were lost to follow up. Four infants (6.3\%) died before surgery could be carried out due to failure to thrive. Two of these latter cases had associated congenital anomalies. More than half (52.4\%) of the patients attended the first review appointment while only $20.6 \%$ of the patients were seen at the third post operative review.

\section{Discussion}

Epidemiological data in the published literature have shown a low prevalence of cleft in Africa compared to Europe, America and Asia $[9,10]$. This low prevalence however may not be the true representation of the burden of orofacial clefts in Africa as most of these studies were retrospective, based on hospital records that may not have accurately reported congenital anomalies $[16,17]$. However, there has been a significant increase in the number of cleft cases presenting and managed at various health facilities across the African continent with increasing public awareness campaigns and sponsorship by many charity organizations such as Smile Train. Over $70 \%$ of the patients in this study presented late for treatment and this is in agreement with previous studies from Nigeria and other developing nations $[14,15,18]$. Although the precise reasons for late presentation have a wide geographical and sociocultural variation, the general factors implicated include lack of funds, ignorance, lack of knowledge of availability of cleft services, long distance from cleft centre, cultural beliefs and fear of surgery $[14,15,17,18]$. A possible explanation for the increasing number of late cleft presentations may be the outcome of advocacy, awareness campaigns, establishment of more cleft treatment centres in both rural and urban areas by governmental and nongovernmental organizations like Smile Train. Studies from other developing nations have also reported similar significant increases in the number of cleft patients seen and managed since the inception of Smile Train activities in their region [19]. It is worth noting that all the cleft patients seen and treated in our centres during the study period were treated free (courtesy of sponsorship by Smile Train organization).

This study showed that unilateral cleft lip with or without cleft palate was the most common orofacial cleft deformity seen in this environment. This is similar to a previous report by Omo-Ahoja et al. [20]. In contrast, most studies in Caucasian and Asian populations have reported a higher frequency of isolated cleft palate [2,21]. Although some of these variations may be attributable to study design, they may also reflect a biological phenomenon. The distribution of cleft types and prevalence may be racially and ethnically determined [22]. This study showed a preponderance of isolated cleft lip in males while isolated cleft palate occurred more commonly in females, in agreement with previous studies [7,11,23-26]. Our report showed that the OFC is more common on the left of the face than the right as previously shown $[27,28]$. The left side of the face was affected twice as often as the right; a similar trend was previously demonstrated by Derijcke et al. [11].

Beside genetic and environmental factors, consanguinity has also 
been implicated in the development of cleft lip and palate and other congenital anomalies $[5,6]$. The risk of associated congenital disorders is about 2 to $3 \%$ in consanguineous marriages and 5 to $8 \%$ in first degree consanguineous marriages [7]. This study failed to find an association of OFC with consanguineous marriage and we feel this may have been due to a relatively small sample size or the cultural practice in Africa. Consanguinity is very rare in African culture making the summation of genetic traits leading to congenital anomalies remote. This view is supported by Iregbulem's study [29].

In contrast to other studies which reported a family history of 10$20 \%$, only $6.3 \%$ of the patients had a positive family history associated with the risk of cleft deformities [20,30]. In addition, the dizygotic twins in our study were discordant for presence of a cleft. This is not unexpected, and has been reported by previous investigators. This trend may suggest an important role of environmental factors in the development of OFC and this had been previously demonstrated by Srivastava and Bang [12].

Many other congenital anomalies have often been found in association with orofacial clefts. Our study showed that $9.5 \%$ of the cleft cases were associated with other congenital anomalies (Table 2). Previous authors have reported a wide range for the frequency of associated anomalies, from $4.3 \%$ to as high as $66 \%$ [31,32]. The wide range of reported frequencies of associated congenital anomalies has been attributed in part to the varying methods of data collection, with lower incidence being reported by studies that have reviewed birth certificates (not birth registries) than studies that account for patients referred to their institutions for treatment [13]. More than two thirds of the congenital anomalies recorded were seen in cleft palate with or without cleft lip while none was recorded in association with isolated cleft lip deformity. Malformations of the musculoskeletal system and the head and face were the most common, with congenital heart disease accounting for the least. This is consistent with previous studies [13].

Limitations of this study include the small sample size and the lack of long term follow up data. There was a high attrition during the follow up period as only about $1 / 5^{\text {th }}$ of our patients attended the third follow up review (one month) after the initial surgery. Lack of long term follow up data is a common phenomenon in Africa and this is multifactorial in cause, including ignorance, poverty (lack of money for transport), long distance to travel for follow up and lack of time.

\section{Conclusion}

Poverty and ignorance are the common causes of late presentation of cleft patients for treatment in this study. Attendance at most cleft treatment centers in Nigeria and indeed in Africa has increased significantly. There is a great need to continue to sustain the availability of cleft lip and palate treatment services to the population through public education and awareness campaigns, provision of treatment centers, facilities and trained multidisciplinary teams. A well designed prospective study on this entity is required in the developing world.

\section{Acknowledgement} advice.

Dr. Ayantunde A. Abiodun for reading the manuscript and offering valuable

\section{References}

1. Edward Ellis III (2003) Contemporary Oral and Maxillofacial Surgery, (4thedn). Mosby Inc. Management of patients with orofacial clefts.

2. Calzolari E, Pierini A, Astolfi G, Bianchi F, Neville AJ, et al. (2007) Associated anomalies in multi-malformed infants with cleft lip and palate: An epidemiologic study of nearly 6 million births in 23 EUROCAT registries. Am J Med Genet A 143: 528-537.
3. Randall P, La Rossa D (1974) Cleft palate. Plastic Surgery (4thedn).

4. Melnick M (1992) Cleft lip (+/- cleft palate) etiology: a search for solutions. Am J Med Genet 42: 10-14.

5. Rittler M, Liascovich R, López-Camelo J, Castilla EE (2001) Parenta consanguinity in specific types of congenital anomalies. Am J Med Genet 102: 36-43.

6. Leite IC, Koifman S (2009) Oral clefts, consanguinity, parental tobacco and alcohol use: a case-control study in Rio de Janeiro, Brazil. Braz Oral Res 23 31-37

7. Kanaan ZM, Mahfouz R, Tamim H (2008) The prevalence of consanguineous marriages in an underserved area in Lebanon and its association with congenital anomalies. Genet Test 12: 367-372.

8. Mossey PA, Little J (2002) Epidemiology of Oral Clefts: An Internationa perspective in Cleft lip and palate. In from origins to treatment. Edited by Wyszynski DF. Oxford University Press.

9. Vanderas AP (1987) Incidence of cleft lip, cleft palate, and cleft lip and palate among races: a review. Cleft Palate J 24: 216-225.

10. Croen LA, Shaw GM, Wasserman CR, Tolarová MM (1998) Racial and ethnic variations in the prevalence of orofacial clefts in California, 1983-1992. Am J Med Genet 79: 42-47.

11. Derijcke A, Eerens A, Carels C (1996) The incidence of oral clefts: a review. Br J Oral Maxillofac Surg 34: 488-494.

12. Srivastava S, Bang RL (1990) Facial clefting in Kuwait and England: a comparative study. Br J Plast Surg 43: 457-462.

13. Elahi MM, Jackson IT, Elahi O, Khan AH, Mubarak F, et al. (2004) Epidemiology of cleft lip and cleft palate in Pakistan. Plast Reconstr Surg 113: 1548-1555.

14. Schwarz R, Bhai Khadka S (2004) Reasons for late presentation of cleft deformity in Nepal. Cleft Palate Craniofac J 41: 199-201.

15. Adeyemo WL, Ogunlewe MO, Desalu I, Ladeinde AL, Mofikoya BO, et al (2009) Cleft deformities in adults and children aged over six years in Nigeria: Reasons for late presentation and management challenges. Clinical Cosmetic and Investigational Dentistry 1: 63-69.

16. Butali A, Mossey PA (2009) Epidemiology of Orofacial clefts in Africa: Methodological challenges in ascertainment. Pan Afr Med J 2: 5.

17. Orkar KS, Ugwu BT, Momoh JT (2002) Cleft lip and palate: the Jos experience. East Afr Med J 79: 510-513.

18. Adesina OA, Olasoji HO, Ugboko VI (2010) Reasons for late presentation of cleft deformity in Northeastern Nigeria. Kanem Journal of Medical Sciences.

19. Singh SK (2009) Smile Train: The ascendancy of cleft care in India. Indian J Plast Surg 42 Suppl: S192-198.

20. Omo-Aghoja VW, Omo-Aghoja LO, Ugboko VI, Obuekwe ON, Saheeb BD, et al. (2010) Antenatal determinants of oro-facial clefts in Southern Nigeria. Afr Health Sci 10: 31-39.

21. Cooper ME, Ratay JS, Marazita ML (2006) Asian oral-facial cleft birth prevalence. Cleft Palate Craniofac J 43: 580-589.

22. Onyango JF, Noah S (2005) Pattern of clefts of the lip and palate managed over a three year period at a Nairobi hospital in Kenya. East Afr Med J 82: 649-651.

23. Adekeye EO, Lavery KM (1985) Cleft lip and palate in Nigerian children and adults: a comparative study. Br J Oral Maxillofac Surg 23: 398-403.

24. Obuekwe O, Akapata O (2004) Pattern of cleft lip and palate [corrected] in Benin City, Nigeria. Cent Afr J Med 50: 65-69.

25. Bellis TH, Wohlgemuth $B$ (1999) The incidence of cleft lip and palate deformities in the south-east of Scotland (1971-1990). Br J Orthod 26: 121-125.

26. FitzPatrick DR, Raine PA, Boorman JG (1994) Facial clefts in the west of Scotland in the period 1980-1984: epidemiology and genetic diagnoses. J Med Genet 31: 126-129.

27. Al Omari F, Al-Omari IK (2004) Cleft lip and palate in Jordan: birth prevalence rate. Cleft Palate Craniofac J 41: 609-612.

28. Freitas JA, Dalben Gda S, Santamaria M Jr, Freitas PZ (2004) Current data on the characterization of oral clefts in Brazil. Braz Oral Res 18: 128-133. 
Citation: Aimiede OS, Olalere GO, Olaosun Adedayo O, Sotannde Adeshola I (2013) Orofacial Clefts: Our Experience in Two Suburban Health Facilities. Dentistry 3: 155. doi:10.4172/2161-1122.1000155

29. Iregbulem LM (1982) The incidence of cleft lip and palate in Nigeria. Cleft Palate J 19: 201-205.

30. Fraser FC (1970) The genetics of cleft lip and cleft palate. Am J Hum Genet 22: $336-352$

31. Jensen BL, Kreiborg S, Dahl E, Fogh-Andersen P (1988) Cleft lip and palate in
Denmark, 1976-1981: epidemiology, variability, and early somatic development. Cleft Palate J 25: 258-269.

32. Altunhan H, Annagur A, Konak M, Ertuqrul S, Ors R, et al. (2012) The incidence of congenital anomalies associated with cleft palate/cleft lip and palate in neonates in the Konya region, Turkey. Br J Oral Maxillofac Surg 50: 541-544. 